\title{
Aortic Surgery Symposium 2012 discussions-Panel 3 (sessions V and VI): Descending thoracic aorta, thoracoabdominal aorta, and trauma
}

\author{
Moderator: Randall B. Griepp, MD, New York, NY. \\ Panelists: Joseph S. Coselli, MD, Texas Heart Institute, Baylor College of Medicine, Houston, Tex; John Elef- \\ teriades, MD, Yale University School of Medicine, New Haven, Conn; Gabriele Di Luozzo, MD, Mount Sinai \\ School of Medicine, New York, NY; Hazim J. Safi, MD, University of Texas Medical School, Houston, Tex; \\ Nicholas T. Kouchoukos, MD, Missouri Baptist Medical Center, St. Louis, Mo; D. Craig Miller, MD, Stanford \\ University School of Medicine, Stanford, Calif; Nicholas J. W. Cheshire, MD, Imperial College Academic \\ Health Science Centre, London, UK; Eric Roselli, MD, Cleveland Clinic, Cleveland, Ohio; Anthony L. Estrera, \\ MD, University of Texas at Houston Medical School, Houston, Tex.
}

\section{Discussion}

Dr Griepp. Tony, may I ask you a question? How difficult do you think it is to sort out in your own experience with aortic trauma, the delayed repair and the advent of thoracic endovascular aortic repair (TEVAR)? Do you think if TEVAR hadn't come along, you would be having these same results with open repair at this point, just by having added the delayed repair for those patients who can have the delay?

Dr Estrera. It is very complicated to sort out. When we published this article a couple of years ago, the only variable that was protective in multivariable analysis was actually delayed repair; TEVAR was not. Now, after having added another 35 cases or so, I have to think that TEVAR might play a role. But it is very difficult.

And I didn't give credit where credit is due, to be honest with you, because a lot of the improvements should take into consideration the trauma team and all the advancements that have occurred in trauma care generally. In aortic trauma, we think about aortic injuries killing the patients. But the reality is that these patients have a lot of other injuries, and there have been a lot of advancements in trauma care during the past decade that also have contributed to better outcomes. I don't think that we should think or say that delayed repair or TEVAR is the only factor, and it is difficult to say that the data really bear out such a claim at this point.

Another point in that original article that has changed now is the fact that in our early experience we delayed repair in many of these patients because they had an abnormal white blood cell count or something else that we feared would cause a problem with graft infections. Now, however, we have reduced the preintervention hospital stay with TEVAR to about 1 day from the time the diagnosis is made. So as soon as that diagnosis is made, we rush the patient up to the hybrid suite, put the TEVAR graft in, and repair the aorta. Then the patient can go back to the trauma service,

\footnotetext{
Disclosures: Authors have nothing to disclose with regard to commercial support. Read at Aortic Surgery Symposium 2012, New York, NY, Thursday, April 26-27, 2012.

Address for reprints: Eva B. Griepp, MD, Department of Cardiothoracic Surgery, Mount Sinai School of Medicine, New York, NY 10029 (E-mail: ebgriepp@aol. com).

J Thorac Cardiovasc Surg 2013;145:S159-64

$0022-5223 / \$ 36.00$

Copyright (c) 2013 by The American Association for Thoracic Surgery

http://dx.doi.org/10.1016/j.jtcvs.2012.11.082
}

and we don't worry about anti-impulse therapy or anything like that.

Dr Griepp. Let me just ask one other question. Dr Kouchoukos, you are perhaps the biggest advocate here of using hypothermic circulatory arrest for thoracoabdominal aortic aneurysms. Most of the concerns expressed by people who are interested in exploring this technique have to do with bleeding problems. So maybe you can tell us just a little bit about how you deal with postoperative bleeding and manage coagulation in these patients.

Dr Kouchoukos. We don't do anything special. I think there has always been a concern about bleeding, but the experience with hypothermic circulatory arrest for ascending aortic disease and arch disease has led us to feel more confident about using it in the descending thoracic aorta. We use hypothermic circulatory arrest exclusively in the descending aorta, and we also use it for thoracoabdominal aneurysms.

I showed you some of the transfusion requirements. We looked at them in general, and if you compare the blood requirements with those for other perfusion techniques that have been used, they do not exceed those requirements. We use fresh-frozen plasma; we use platelets; we use cryoprecipitate. We have only used factor VII in 1 or 2 cases in which the bleeding was extensive. So obviously we screen these people carefully preoperatively to be certain that they don't have coagulation problems, but bleeding has not in general been a big problem.

Dr Griepp. Do you have a routine of what you give at the end of the operation, or does it depend on each patient?

Dr Kouchoukos. We have operated on several Jehovah's Witnesses to whom we haven't given any blood products. We give blood products on an as-needed basis. If the perfusion time has been longer than 2 hours, we prophylactically give platelets and fresh-frozen plasma, and occasionally cryoprecipitate, at the completion of the procedure.

Dr Manu N. Mathur (St Leonards, New South Wales, Australia). This is a question for Professors Elefteriades and Sundt. Is there any evidence at all for replacing the ascending aorta in patients with bicuspid valves when the bicuspid valve is fine, the root is fine, and the ascending aortic aneurysm is smaller than $5 \mathrm{~cm}$ ?

Dr Elefteriades. In all our analyses, we have taken the biggest diameter of the aorta at any level, at the belly of the aneurysm if you will. The management of an aneurysm in the ascending aorta in a patient with a bicuspid valve can be decided on the basis of the largest diameter at any level. Our studies at Yale have found that the behavior of the ascending aorta in the patient with a bicuspid 
valve is not very different from that of the ascending aorta in the patient without a bicuspid valve. If you are getting into questions of what to do if you are going in for the valve, or if you are going in for the aorta, the questions of what should be done for the valve and so on become more complicated. I think that most surgeons would replace the aorta when they are going in for the valve when the aorta is larger than $4.5 \mathrm{~cm}$ in diameter, and some when it is larger than $4 \mathrm{~cm}$.

Dr Leonardo Esteve Lima (Brasilia, Brazil). I have to congratulate Dr Roselli, and I have a question. We believe that technology evolves and that we need expanding options, as you said. After starting to use the Cook device, we switched to the Gore Excluder C3 device for thoracoabdominal aneurysms, with a fenestrated branch. It is faster to use and easier to maneuver. With this kind of friendly device, we do believe that we can use it even for emergency cases. We believe that the options have been expanded, and one can use devices for more cases. What is your opinion about that?

Dr Roselli. I don't have any experience with that device. I think that we are going to see all these devices becoming more valuable. We have brought the Endologix device to our institution and have been using that, but for elective cases. We have branch devices for the arch that we have been using. I don't have any experience with the Gore fenestrating device, but I think as long as you are following these patients up and you are confident that these repairs are durable, it's great. We study our experience closely, and there is room for a lot of advancement.

Dr Lima. Just to give you an example, we took about an hour and a half for an emergency trifurcated branch. That is very fast compared to the Cook device, and so that's why we don't use the Cook in some emergencies. What do you think about the time?

Dr Roselli. Well, for a 3-branch device in an abdominal aneurysm, I saw Ed Bevins fix a rupture of a juxtarenal aneurysm in 15 minutes. So your hour and a half sounds long. But you have to look at the question on a patient-to-patient basis. I think it is wonderful if you can get good results with these devices. The important thing is to understand the limitations of all of them and to be comfortable with the particular device that you use.

Dr Jeffrey Gibson (Germantown, Tenn). Anthony, can you tell me a little bit about how you manage your delayed patients medically and how you "cooperate" (I guess that is a good word) with the vascular surgeons in caring for these patients?

Dr Estrera. A good question. The way our department is set up is that the vascular surgeons are also the cardiac surgeons and the thoracic surgeons. So we all work together, and we discuss all these cases. So it is not really an issue in our department, because we have one head-Hazim Safi-and he directs everything else.

But with regard to the trauma service, it's a good question. It has almost become a moot point, to be frank with you. When we used to delay these patients, and put the stents in later, cooperation was an issue, and it was a complicated issue. The trauma service, especially in head injury, would want to maintain the pressures higher, and we were trying to get the pressures down and administering a $\beta$-blocker or anti-impulse therapy. It was very complicated at that time.

What has simplified everything is that as soon as the patient gets in and the diagnosis of aortic trauma is made, the patient is run up to the operating room (the hybrid suite), and we deploy the stent and fix the traumatic injury. And then the patient goes to the shock trauma intensive care unit and is managed there, and we don't limit the blood pressure at that point. In fact, with our TEVAR experience, we don't mind higher pressures, especially to prevent spinal cord complications. So the advent of TEVAR has decreased our use of delayed selective management at this point.

Mr Cheshire. Tony, can you just mention, with that policy, whether you have had any acute graft collapses or failures?

Dr Estrera. The short answer is no. Again, we are very selective, and we have to make sure that we have good anatomic boundaries to put in the stent. Ali Azizzadeh, Kristofer Charlton-Ouw, and Sheila Coogan are our vascular surgeons. We work together, and these cases we discuss every time they occur and make decisions together.

I can justify our policy of using devices in my mind because we have seen a decrease in early mortality. I think I have done 1 open repair in the last 3 years at our institution for traumatic aortic rupture, because we just take the patients straight to the hybrid suite for stent placement. This possibility has really changed how we think about it. But, as Craig Miller said earlier, we need longterm results, and the article that he presented on aneurysms is very sobering. If there are any differences in mortality at 5 and 10 years, then I will rethink things. I can justify immediate stent placement now, however, because we have shown a decrease in the early mortality in this situation.

Dr Miller. Tony, I'm confused; help me. You just answered the gentleman's question by saying that TEVAR has now supplanted your delayed permissive hypotension treatment. In the last year or so, how many patients are undergoing TEVAR without thinking, as is happening almost everywhere around the world? During the last year or so, have you been doing anything except TEVAR?

Dr Estrera. In the last 3 years, we have done 1 case open. So we have done everything with TEVAR; we do TEVAR immediately after the injury. TEVAR has thus supplanted delayed selective management. When we were doing the repairs open, we used to delay these patients and get them in a little bit better physiologic state if they didn't have grade 4 ruptures or even severe grade 3 ruptures with large hematomas. The ones with ruptures or large hematomas, I'd operate on immediately.

Dr Miller. So the tide has swept you out with it?

Dr Estrera. It did when we analyzed these data. As Thor Sundt said 6 years ago at this meeting about the denominator, we as surgeons live in the numerator: we only see what we do. We looked at our overall admissions for traumatic aortic rupture, however, and I saw that the mortality had decreased. I know it is not just the use of TEVAR early, but the improving early results did make a difference in my mind. At this point, I accept the early use of TEVAR because I think it helps in the initial postoperative period. But 5 to 10 years out, if we start to see problems in a 20 - or 30-year-old, I will rethink our ideas and change.

Dr Miller. Following up, didn't you tell us that the multivariate analysis showed that delayed surgical repair was protective in terms of mortality, but TEVAR was not?

Dr Estrera. That's correct.

Dr Miller. I hear some hypocrisy here.

Dr Estrera. That's correct, yes.

Dr Miller. You are going to have a hard time following these patients up, because, as you know, the best follow-up of any acute 
traumatic tear series is $40 \%$ to $50 \%$. You have check with Interpol, the border patrol, the Drug Enforcement Agency, US Immigration and Customs Enforcement. We just can't follow these young folks up.

Dr Estrera. But Craig, we looked at every patient, and with the way we are managing them now, the mortality has decreased. It's hard for me, because these patients-as you know-are very sick. They come in, and you do a big open operation, which is a good technical operation but more invasive than TEVAR. These patients are physiologically insulted: they have liver injury, they have splenic injury, they have head injury, and they don't do as well as our elective descending thoracic aneurysm repair. They just don't. So I have justified early TEVAR in my mind, but I don't disagree with what you are saying. In the end, if we see a decrease in long-term survival with TEVAR, then we will switch back to delayed open repair.

Dr Miller. But the delayed treatment with an open surgical procedure 1,2 , or 3 weeks later, that's definitive and it is low risk, correct?

Dr Estrera. Potentially.

Dr Griepp. I'm going to let Mr Cheshire have the final comment.

Mr Cheshire. I was just going to comment on being swept by the wave and Dr Miller's comments. In the United Kingdom, the national data would suggest that only about $20 \%$ of thoracic aneurysms are now repaired open: that ship has almost sailed from our country.

Dr Griepp. Interesting. Let me just move to a slightly different subject.

Drs Safi and Coselli have both published a lot of data having to do with the impact of the status of renal function on operative mortality and complications. I ask each of you, have you thought at all about why this is and whether there is any way to change that when you have got such a great marker? How do you move somebody from a $30 \%$ risk to a $10 \%$ risk? Is it just a marker for poor protoplasm?

Dr Safi. Well, we looked at it, and, as you said, it's a marker. I don't know what the impact of our protection of the kidney is going to achieve. Dr Tray Miller is interested in that subject. But apart from that, I think that if we can eliminate renal failure postoperatively, we make our operation better than the endovascular.

Dr Coselli. We haven't looked at glomerular filtration rate (GFR) the same way that Drs Estrera and Safi have, but I also think it's almost certainly a marker. One of the things that backs that up is that we have seen many fewer type 1 aneurysms because of endovascular work. We are still doing a sizable number of extent 2 aneurysms, but the really high-risk patients that we are facing now are the elderly patients with type 3 and 4 aneurysms with an enormous amount of superimposed atherosclerosis, who frequently start with minimal or no renal function. I think that blends into that whole process as an evolution of the risk profile, but we haven't really culled it out the way they have.

Dr Griepp. Do you think that all the renal dysfunction that you are measuring as a risk factor is from atheroemboli or from hypertension? Why are these patients so different?

Dr Coselli. I think it's a combination of both. We think it's superimposed atherosclerosis. Many of these patients with type 3 and 4 lesions only have one kidney. Then there is another group of patients, which overlaps somewhat, who come in with extremely poor renal function but decent anatomy and have hypertensive nephropathy so that their GFR is really quite low. But the etiology of the hypertensive nephropathy has affected their organ function elsewhere: not only has it impacted the kidneys, it has increased the risks of stroke, myocardial infarction, and multiple organ failure.

Dr Safi. When Dr Tray Miller looked into our thoracoabdominal and descending aneurysms, the patients with descending aneurysms have the best GFR. It is not normal, but it is $75 \mathrm{~mL} /$ min. The worst GFR is with extent 4 aneurysms. And once the viscera are involved with atheromatous plaque and aneurysm, kidney function deteriorates. Probably, as Joe said, it is a combination. I think that microemboli go there and kill the kidneys. In the literature, they say that extent 4 is best suited for stenting. They can have these cases, with their low GFR. The average, in our experience, is almost $50 \mathrm{~mL} / \mathrm{min}$, and these cases have the highest mortality.

Dr Ziad Hanhan (Jersey City, NJ). I have questions for Drs Safi and Estrera. If you gentlemen, God forbid, had a traumatic aortic rupture-isolated-how would you have that addressed for yourself?

Dr Safi. If it comes to myself, I know what he is going to do. I tell him to go and cut me and put a graft in, and I'm done. But I'm sure he is going to cut my vocal cords so I can't talk to him.

Dr Estrera. That's a great question. Isolated, no other injury, and Craig Miller will love to hear this, I would have a good surgeon-Dr Safi's vocal cords don't work anymore-so I would have a good surgeon cut me open and just fix it so I'm done. I don't need any radiographs or computed tomographic (CT) scans or anything for the rest of my life.

Dr Jehangir Appoo (Calgary, Alberta, Canada). I wanted to ask Dr Elefteriades whether he has been able to extrapolate the bench engineering work with strain and stress to some sort of in vivo model to assess aortic wall strain and stress on CT to help us clarify what has been discussed a lot today: which patients should be operated on, and which patients shouldn't, on the basis of something more specific than diameter.

Dr Elefteriades. You bring up an absolutely superb point. Diameter is good, but there has got to be more, and I think genes and engineering are the more that we want to achieve. And there are efforts under way. I was talking with Pria from Papworth about this and some others also. There are efforts underway to quantitate wall stress based on CT scan or magnetic resonance imaging. I think that it is important to realize that when you get down to looking at those equations, there are assumptions that are made that may not be realistic or may limit accuracy.

One such assumption is the blood pressure. The blood pressure is checked by cuff sometime before the examination is done, and then it is presumed to be steady during the examination. We all know from the operating room that this is not true. And then, if I am correct, most of the equations assume the elastic modulus of the patient's aortic tissue to be the average elastic modulus known from studies like ours and those done elsewhere. So there are those important assumptions that are made. All those very fancy equations look as though they would be terrific, but, again, I think they are assuming the constant blood pressure and they are assuming the average elastic modulus. 
With all that said and done and taken into account, I still think that the in vivo assessment of wall stress in individual patients, along with the genetics that Dr Maleic talked about yesterday, will be the next milestones in our decision making regarding when to operate. This is a very important point; thank you.

Dr Said A. B. Silliman (Cairo, Egypt). My question is for Drs Safi and Kouchoukos about reimplanting the lower intercostals and the first lumbar intersegmental artery. You mentioned 2 techniques: circulatory arrest with deep hypothermia for Dr Kouchoukos, and the other technique, used by Dr Safi, without circulatory arrest. I noticed that Dr Safi pays attention to reimplanting the lower intercostals. What does Dr Kouchoukos say with regard to reimplantation?

Dr Kouchoukos. We implant the lower lumbar intersegmental arteries when they are patent. We are more inclined to do it in patients with chronic dissection, particularly in the younger patients who have extensive aneurysms. With respect to the patients who have severe atherosclerotic disease-the patients Dr Safi was talking about, those with extent 3 and 4 disease with extensive atherosclerosis-it is rare, in our experience, that you find patent intercostal arteries, at least lower intercostal arteries and lumbars that can be implanted. And Dr Griepp has challenged the notion that you need to implant them at all.

In our analysis of our cases, we found no difference in the prevalence of paraplegia between the patients in whom we implanted intercostal arteries and lumbars and those in whom we didn't. And I think among the reasons may be that if you look at these patients with follow-up CTs, many times these vessels are not open after they have been reimplanted. So I am not sure that we really know the answer.

Dr Safi. Well, in the past we reimplanted them in anyone, from T8 to T12. In the last 5 years, I think that Tony Estrera has changed the practice in our work. Tony, will you tell them how you are using neuromonitoring?

Dr Estrera. We presented this 2 years ago at this meeting, a kind of neuromonitoring-guided thoracoabdominal replacement. In that protocol, we use motor-evoked potentials as well as somatosensory-evoked potentials. If we get no change during surgery at the time where we would be working on those intercostal arteries, we don't reimplant them. If we don't ever have a change, we just ligate them.

When we looked at the data, I think it was from more than 100 cases, it turns out that in $30 \%$ of the cases that you could reimplant $\mathrm{T} 8$ to L1, implanting the intercostal arteries allowed us to recover our motor-evoked potentials. So about $30 \%$ of the time it is beneficial to reattach intercostal arteries in the way we do our operation.

Now, Dr Griepp ligates all intercostal arteries before the aorta is clamped. So how he does the operation-his model-is a little bit different. But the way we do the operation, intercostal artery reattachment was beneficial $30 \%$ of the time. So it has been eliminated $70 \%$ of the time, and decreased our operative time. But the other change we have made concurrently is to use a loop graft to reattach the intercostal arteries as opposed to an inclusion patch; we saw a number of intercostal patch aneurysms in the past.

Dr Kouchoukos. Has your new method reduced the prevalence of paraplegia?
Dr Estrera. No, not significantly from our previous experience. We didn't have enough cases to answer the question. Dr Safi showed a slide from the article that was published, and the incidence of paraplegia was about $2 \%$. This is a problem with all studies of paraplegia now: the incidence is fairly low. So you are going to need a large number of cases to show any difference. The neuromonitoring protocol has decreased our overall operative time however, in that we don't have to reattach so many intercostal arteries.

Dr Di Luozzo. I just wanted to make a comment. Eric Roselli doesn't implant any intercostals when he does hybrid thoracoabdominal cases. So where is the validity of implanting in the open cases? In our protocol, and as we move on with endovascular repairs, there won't be any reimplantation of intercostal arteries, and the evidence is that the patients have a very low incidence of spinal cord injury.

Mr Cheshire. It probably is worth mentioning some of these newer techniques, though, for example very long aortic coverage with stent-grafts that leave a single sac perfusion branch open, allowing the patient to settle for a couple of weeks, and then the perfusion branch is closed either permanently or as a trial, and the spinal cord monitored. Because-as Dr Griepp and I have discussed, previously - I think that this is an idea that follows directly from his work of understanding the serial nature of closing off intercostals, even though Krassi Ivancev, who invented it, claims he didn't even know that work but just came up with the idea. It's very valid, however, and I could see that allowing time between ligation of intercostals might have a real future.

Dr Roselli. If I could expand on my remarks, although it's true that we don't reimplant intercostals with endovascular repairs, we do look at the patient's entire vascular collateral supply to the spinal cord when we assess these patients. There are patients in whom we feel that the intercostal bed is really important because they have compromised hypogastrics or something of that sort, and those patients we will not treat with an endovascular operation but will send them for open surgery. And so I think, once again, you have to assess the situation on a patient-by-patient basis.

Dr Coselli. One interesting thing is the issue of delayed paraplegia. We have in the last 600 cases or so seen a flip. Before, we would see about two thirds immediate paraplegia versus a third delayed paraplegia; now, it's almost the opposite. We are seeing about two thirds delayed and a third immediate, just stressing the importance of some of the factors involved with postoperative care.

We still reattach intercostal arteries. We had an experience with somewhere around 100 patients in which we used motor-evoked potentials, trying to duplicate what Jacobs has described, but we had so many false negatives and false positives that it really didn't affect or alter our results enough to continue to use motor-evoked potentials. If you do use them, there are some issues with anesthesia; there are some concerns with lower extremity ischemia that affect your readings and results, and it's not very adaptable to deep hypothermic cases.

Dr Griepp. I will just add a final comment. One thing that pretty much everyone can agree on is the importance of the hypogastric arteries in the spinal cord circulation. As I said before, I think that much of the data having to do with the risk factor for paraplegia with TEVAR and the thoracic aorta with previous abdominal aortic repair probably had very little to do with the lumbar 
segmental vessels but rather had to do with sacrificing the internal iliacs. I think all of us probably appreciate that as one treats the thoracoabdominal aorta, closing off the hypogastrics is not a benign procedure. They are probably some of the most important collateral pathways for spinal cord blood supply.

Dr Michael T. Janusz (Vancouver, British Columbia, Canada). I have a question for Drs Safi and Kouchoukos regarding interposition grafts for the visceral vessels. Through the years, I have quite often used an interposition Dacron polyester fabric graft to the left renal in a thoracoabdominal aneurysm repair, with direct implantation of the other vessels. In the first few years of CT follow-up, the patency of the interposition graft was fine, but in later films taken 10 years or more postoperatively, it is not uncommon to see occlusion of that Dacron graft. I don't have any numeric data on it, but whenever I do see that, I am thankful that I didn't use an interposition graft on the other renal. Do you have any information about the long-term follow-up of interposition grafts in this position?

Dr Kouchoukos. We looked at a series of these patients that we published several years ago, for whom we have follow-up of at least 5 years. In the time since, we have followed these patients up fairly regularly with imaging, and we have only seen three patients who developed either stenoses or occlusions. The stenoses were treated with stents. The one patient with occlusion had some critical event where he became hypotensive and thrombosed all the branches. In general, the grafts have stayed patent in our experience, and we haven't seen to date any late occlusions. Obviously there is attrition in this population of elderly patients: they may die and we may not know about these occlusions. But at least among the ones we have followed up, we have not seen any significant occlusions.

Dr Safi. We limit the use of side-arm grafts to patients who are young and have connective tissue disorders. When we need to do it in a patient with medial degenerative disease, to the renal arteries, I tend to use a bigger graft, not 8; I use 10 or 12 . That is what I learned from Dr Crawford. And we didn't have a problem, as you said.

Dr Griepp. I would add to that. In Dr Spielvogel's series of trifurcation grafts to the arch vessels, I think it is true that we have yet to see an occluded arch branch graft in annual postoperative CT scans. Is that still right, David?

Dr David Spielvogel (Valhalla, $N Y$ ). As far as I know.

Dr Griepp. And almost all of these patients have annual CTs in our follow-up program. So at least in the arch, occlusion of a branched graft is extremely unusual.

Dr Vicki Mahan (Worcester, Mass). My question is directed to all the panel members. What are the indications for TEVAR in the patient with Marfan syndrome?

Dr Griepp. Craig, you will have fun with this one.

Dr Miller. That's a very easy question. None.

Dr Coselli. Dr Miller is referring to TEVAR as a primary definitive procedure in Marfan syndrome. But we have used endovascular repair in some intercostal patch aneurysms, in which basically you are relining the graft. We have had some patients with extent 4 disease who have had some previous grafts either in the arch or the very proximal descending in whom we have used TEVAR graft to graft. Also, I think that you have to give some consideration to lifesaving emergency situations as a bridge, again, depending on your skills and environment.
Dr Elefteriades. I am strongly biased toward open repair even in the patient without Marfan syndrome, so I am not a good one to comment on that.

Dr Safi. With me, the answer is none. There is no reason to put in a stent in a patient with a connective tissue disorder.

Dr Kouchoukos. I would be to the right of Craig Miller on this one as well. The people who have tried it have had problems with introduction of retrograde dissections. There is a recent publication from the University of Florida in which they looked at a group of patients with Marfan syndrome, and the complication rate was extraordinary. I think that article provides a good lesson about why not to do it in patients who have these connective tissue or genetically determined disorders.

Dr Di Luozzo. I agree with Dr Coselli. I think that in emergency situations it is a good bailout, and also if you have a case where you have a graft to graft situation, if patients have had previous surgery. Otherwise, in elective cases, perform open surgery.

Mr Cheshire. We think that there are no indications, but we have done about 12 in our series either because the patient was very sick at the time or because there were other contraindications to open surgery. Occasionally, you can get away with it. We haven't seen any retrograde dissections, either.

Dr Roselli. I am going to echo what most of the panel have said, except for the none part. I think that there is definitely an indication for stent-grafts in patients with Marfan syndrome, because there is a certain spectrum of aortic degeneration that we see in these patients, and the ones who have the entire aorta degenerated often need a lifetime of operations until the whole aorta has been replaced. So I have a whole group of these patients who have undergone what we call an ultrahybrid reconstruction, where they have Dacron polyester fabric grafts sutured into parts of their aorta and stent-grafts interposed, whether it is for a patch aneurysm or some other sort of disaster. But we try to put the stent-graft into Dacron.

Dr Kouchoukos. If you can anchor the stent to a graft, I see no problem there.

Dr Roselli. That is where we have a bunch of cases.

Dr Estrera. I would add none for elective cases, and I do agree with Eric and Gabe and Joe with regard to an emergency situation. I think it would be good in an emergency, lifesaving situation, especially aortoesophageal fistula or aortobronchial fistula: the patient is bleeding, the patient is dying, put a stent there, allow the patient to recover, and then come back in a month or so and take it out and do the definitive operation at that time.

Dr Griepp. So I think the answer is that stents are appropriate only in the part of the patient with Marfan syndrome that is unaffected by Marfan disease, or when it's a temporary procedure. Dr Estrera has to do a certain number of these cases so Dr Safi can make his films of pulling the stents out.

Dr Santi Trimarchi (San Donato, Italy). My question is for Dr Elefteriades. I very much enjoyed your presentation. It was fantastic. It looks as though we have a kind of pocket list that we can perhaps use for the near future to treat very specific patients to prevent rupture and dissection. We are trying to do a similar study as you showed with the engineers on the descending section on both segments, ascending versus descending, and the situation with the descending looks a little bit different. The thickness that we usually have in the descending aorta samples is very different than in the ascending aorta: there are fewer elastic fibers, the presence of 
plaque, and other differences. So what do you think about where we are for descending disease in terms of genes? We don't have very specific genes for descending aortic disease. We don't have much news about specific medical therapy for descending aortic disease. And also, these types of functional and anatomic studies look a little bit different. Can you tell us something more?

Dr Elefteriades. Yes, Santi. I think it is clear that it is really 2 different diseases. From the annulus to the ligamentum arteriosum, it is a noncalcified, heavily genetic disease without thrombus. From the ligamentum distally, it is a different disease. It is calcified, arteriosclerotic, there is lots of thrombus, and the thoracic aorta looks just like the abdominal aorta in that regard. We are beginning to recognize now that the embryologic origins of the ascending and the descending aorta are completely different. So maybe that's part of the reason for the differences.

I think that you are wise to focus on the mechanical differences in those 2 segments. We have to remember that the aorta as it branches is like a tree as it gives off its branches and gets taller. The aorta doesn't need to be as strong more distally along its length. The ascending aorta has 78 lamellae, and the descending aorta has about 28 lamellae. So it is a completely different kettle of fish. I think that you raise a very valuable point, that the 2 aortas are different, and thus we can expect their engineering characteristics to be different, and I applaud and encourage you to explore the mechanical characteristics of the descending aorta.

Dr Pasquale Totaro (Milan, Italy). I have a question for Dr Estrera and, of course, all the other doctors. Do you treat acutely even a traumatic grade 1 or intimal tear, or how do you manage it if you follow up just with medical therapy?

Dr Estrera. It is a difficult subset of patients, because these are the ones we don't put stents in. And again, remember, these are all shadows; ultrasonography, angiography, and CT are all shadows. Trying to interpret what happened in that aorta, you see a little bit of a flap; we call it an intimal tear, we call it a grade 1 injury. We will treat those medically. But they need to be followed up closely: there was 1 patient in the series who came back about a month later with a false aneurysm. So they have to be followed up. And that is really the challenge of this subset of patients, because I don't think that they all need to be treated. I think you need to follow up them and treat them expectantly in case a problem occurs. Following these patients up is difficult, however, especially with TEVAR. As I noted in this presentation, we had complete radiologic follow-up for only $32 \%$ of the patients who underwent TEVAR. 